\title{
On Strong Subadditivity of the Entanglement of Formation
}

\author{
Koenraad M.R. Audenaert, Samuel L. Braunstein \\ University of Wales, Bangor \\ School of Informatics \\ Bangor (Gwynedd) LL57 1UT, Wales \\ E-mail: kauden@informatics.bangor.ac.uk \\ October 17, 2018
}

\begin{abstract}
We employ a basic formalism from convex analysis to show a simple relation between the entanglement of formation $E_{F}$ and the conjugate function $E^{*}$ of the entanglement function $E(\rho)=S\left(\operatorname{Tr}_{A} \rho\right)$. We then consider the conjectured strong superadditivity of the entanglement of formation $E_{F}(\rho) \geq$ $E_{F}\left(\rho_{I}\right)+E_{F}\left(\rho_{I I}\right)$, where $\rho_{I}$ and $\rho_{I I}$ are the reductions of $\rho$ to the different Hilbert space copies, and prove that it is equivalent with subadditivity of $E^{*}$. Furthermore, we show that strong superadditivity would follow from multiplicativity of the maximal channel output purity for quantum filtering operations, when purity is measured by Schatten $p$-norms for $p$ tending to 1 .
\end{abstract}

\section{Introduction}

One of the central quantities in quantum information theory is the entanglement cost of a state, defined as the number of maximally entangled pairs (singlets) required to prepare this state in an asymptotic way. Calculating the entanglement cost of a general mixed state as such is, with the present state of knowledge, a formidable task because one has to consider an infinite supply of singlets and construct a protocol using local or classical (LOCC) operations only, such that the resulting (infinite-dimensional) state approximates an infinite supply of the required state to arbitrary precision. Furthermore, the protocol must have maximal yield, the number of states produced per singlet. The entanglement cost is the inverse of this yield.

An important theoretical breakthrough was achieved in [1, where the entanglement cost $E_{C}$ was shown to be equal to the regularised entanglement of formation: $E_{C}(\rho)=\lim _{n \rightarrow \infty} E_{F}\left(\rho^{\otimes n}\right) / n$. The entanglement of formation (EoF) (defined below in (II) is defined in a mathematical and non-operational way and is therefore much more amenable to calculation. Moreover, for 2-qubit mixed 
states, a closed formula for the EoF exists [2]. Nevertheless, calculating the entanglement cost still requires calculations over infinite-dimensional states. For that reason one would hope for the additivity property to hold for the EoF: $E_{F}\left(\rho_{1} \otimes \rho_{2}\right)=? E_{F}\left(\rho_{1}\right)+E_{F}\left(\rho_{2}\right)$, because then $E_{C}=E_{F}$. Additivity of the $\mathrm{EoF}$ has been proven in specific instances $3,4,5,6,7,8$. Some of these additivity results are sufficiently powerful to allow calculating the entanglement cost for certain classes of mixed states [5,6].7. The much sought-after general proof, however, remains elusive for the time being and, in fact, general additivity is still a conjecture.

It is very easy to show that the EoF is subadditive:

$$
E_{F}\left(\rho_{1} \otimes \rho_{2}\right) \leq E_{F}\left(\rho_{1}\right)+E_{F}\left(\rho_{2}\right) .
$$

Additivity would then follow from superadditivity:

$$
E_{F}\left(\rho_{1} \otimes \rho_{2}\right) \geq ? E_{F}\left(\rho_{1}\right)+E_{F}\left(\rho_{2}\right) .
$$

In 4] a stronger property, which would imply (super)additivity, has been conjectured for the EoF, namely strong superadditivity:

$$
E_{F}(\rho) \geq ? E_{F}\left(\rho_{I}\right)+E_{F}\left(\rho_{I I}\right),
$$

where $\rho$ is a general state over a duplicated Hilbert space and $\rho_{I}$ and $\rho_{I I}$ are its reductions to the different copies of that space.

In this paper we show that strong superadditivity of EoF is equivalent to subadditivity of a much simpler quantity, the so-called conjugate of the entanglement functional $E(\rho)=S\left(\operatorname{Tr}_{A} \rho\right)$. We then exploit this equivalence to show that strong superadditivity would follow as a consequence of multiplicativity of the maximal output purity, measured by a Schatten norm, for quantum filtering operations (this quantity will also be defined in due course).

The main results are stated in Theorems 1 and 2. To arrive at these results, we have made use of a basic formalism from convex analysis [9,10] and we hope that our results will stimulate usage of this elegant theory in other areas of quantum information.

\section{Notations}

Let us first introduce the basic notations. Let $S(\rho)$ denote the von Neumann entropy $S(\rho)=-\operatorname{Tr} \rho \ln \rho$. For state vectors we will typically use lowercase Greek letters, $\psi, \phi$, and assign the corresponding uppercase letter to the projector of that vector; e.g. $\Psi=|\psi\rangle\langle\psi|$. For mixed states we will use lowercase Greek letters $\rho, \sigma, \tau$. The identity matrix will be denoted by $\mathbb{I}$.

We shall denote the set of bounded Hermitian operators over the Hilbert space $\mathcal{H}$ by $\mathcal{B}^{s}(\mathcal{H})$, the set of non-negative elements in $\mathcal{B}^{s}(\mathcal{H})$ by $\mathcal{B}^{+}(\mathcal{H})$, and the (convex) set of all states (trace 1 positive operators) over $\mathcal{H}$ by $\mathcal{S}(\mathcal{H})$.

We will frequently slim down expressions like $\max _{\rho \in \mathcal{S}}\{\ldots\}$ to $\max _{\rho}\{\ldots\}$. When the domain of, say, a maximisation over states is missing it will be implicitly understood that the whole of state space $\mathcal{S}(\mathcal{H})$ is meant. The abovementioned naming convention for states and vectors will be adhered to exactly for that reason. 
Any state $\rho$ can be realised by an ensemble of pure states. An ensemble is specified by a set of pairs $\left\{\left(p_{i}, \psi_{i}\right)\right\}_{i=1}^{N}$, consisting of $N$ state vectors $\psi_{i}$ and associated statistical weights $p_{i}$ (with $p_{i} \geq 0$ and $\sum_{i} p_{i}=1$ ). Here, $N$ is called the cardinality of the ensemble. The entanglement of formation $(\mathrm{EoF})$ of a bipartite state $\rho$ (i.e., a state over the bi-partite Hilbert space $\mathcal{H}_{A} \otimes \mathcal{H}_{B}$ ), is defined by [11]

$$
E_{F}(\rho)=\min _{\left\{\left(p_{i}, \psi_{i}\right)\right\}}\left\{\sum_{i} p_{i} S\left(\operatorname{Tr}_{B} \Psi_{i}\right): \sum_{i} p_{i} \Psi_{i}=\rho\right\}
$$

\section{Convex Closures}

Admittedly, the definition of the EoF just mentioned is not very handy to work with. Not in the least because for generic states $\rho$ the cardinality $N$ of the optimal realising ensemble must be larger than $R^{1.5} / 4$, where $R$ is the rank of $\rho$ [12. This is one of the reasons why no really efficient numerical algorithms have been found yet to calculate the EoF [13]. Furthermore, the mere fact that the minimisation involves ensembles at all makes a theoretical study of the EoF rather difficult. One of the first attempts at proving additivity of EoF relied on the investigation of these optimal ensembles [3].

The results in the present work depend on the following simple observation. The import of the definition (10) of the EoF, as has been shown in 14 4], is that the EoF is the convex closure (or convex roof, as it is called in [14]) of the pure state entanglement function $E(\Psi)=S\left(\operatorname{Tr}_{A} \Psi\right)$, restricted to the set of pure states. This means that the epigraph of the EoF (being the set of points $(\rho, x)$ in $\mathcal{S}(\mathcal{H}) \times \mathbb{R}$ with $\left.x \geq E_{F}(\rho)\right)$ on the complete state space $\mathcal{S}(\mathcal{H})$ is the convex closure of the epigraph of the function $E^{\prime}$ defined over $\mathcal{S}(\mathcal{H})$, where

$$
E^{\prime}(\rho)=\left\{\begin{array}{l}
E(\rho), \rho \text { pure } \\
+\infty, \rho \text { not pure }
\end{array}\right.
$$

This follows immediately from Cor. 17.1.5 of 9] and the definition (11). Note now that $E$ is concave over its domain. There is, therefore, no need to explicitly exclude mixed states ${ }^{1}$, so $E_{F}$ is the convex closure of $E$ as well.

In the following paragraphs we will apply the standard convex analytical formalism for convex closures to general bounded functions $f$ whose domain is the convex set of states $\mathcal{S}(\mathcal{H})$. We will denote the convex closure of $f$ by $\hat{f}$. One definition of the convex closure of $f$ is

$$
\hat{f}(\rho)=\min _{\left\{\left(p_{i}, \rho_{i}\right)\right\}}\left\{\sum_{i} p_{i} f\left(\rho_{i}\right): \sum_{i} p_{i} \rho_{i}=\rho\right\}
$$

agreeing, indeed, with the definition of the EoF. A less cumbersome formulation of the convex closure is based on Cor. 12.1.1 of [9], which states that the convex

\footnotetext{
1 Of course, $E(\rho)$ has no real physical significance for mixed states. Moreover, we must be careful to distinguish between the two possible definitions $E(\rho)=S\left(\operatorname{Tr}_{A} \rho\right)$ and $E^{\prime}(\rho)=$ $S\left(\operatorname{Tr}_{B} \rho\right)$. On pure states, these two definitions yield the same value, but for mixed states this is not so anymore.
} 
closure of a function $f$ is the pointwise supremum of the collection of all affine functions on $\mathcal{S}(\mathcal{H})$ majorised by $f$. So, for all states $\rho$ :

$$
\hat{f}(\rho)=\sup _{X \in \mathcal{B}^{s}(\mathcal{H})}\{\operatorname{Tr} \rho X:(\forall \psi \in \mathcal{H}: \operatorname{Tr} \Psi X \leq f(\Psi))\} .
$$

The mentioned affine functions are here the functions $\operatorname{Tr} \Psi X$, where $X$ ranges over $\mathcal{B}^{s}(\mathcal{H})^{2}$. This dual formulation is then further simplified by defining an intermediate function $f^{*}$ :

$$
f^{*}(X)=\max _{\rho \in \mathcal{S}(\mathcal{H})} \operatorname{Tr}[\rho X]-f(\rho),
$$

the so-called conjugate function of $f[9]$. If $f$ is continuous, then the conjugate function is just the Legendre transform of $f$. The conjugate function is convex in $X$, because it is a pointwise maximum of functions that are affine in $X$. The importance of the conjugate function is that the conjugate of the conjugate of $f$ is the convex closure of $f, \hat{f}=f^{* *}$, and the conjugate of the convex closure of $f$ is the conjugate of $f, \hat{f}^{*}=f^{*}$ (包, the remark just before its Theorem 12.2). Thus

$$
\begin{aligned}
\hat{f}(\rho) & =\max _{X \in \mathcal{B}^{s}(\mathcal{H})} \operatorname{Tr}[\rho X]-f^{*}(X) \\
f^{*}(X) & =\max _{\rho \in \mathcal{S}(\mathcal{H})} \operatorname{Tr}[\rho X]-\hat{f}(\rho) .
\end{aligned}
$$

In other words, the conjugate and convex closure determine each other completely.

Because $f^{*}$ and $\hat{f}$ are convex functions, the optimal $X$ and $\rho$ in (5) and (6), respectively, both form convex sets (possibly singleton sets). Furthermore, there is a correspondence between the optimal $X$ in (5) and the optimal $\rho$ in (6).

Proposition 1. (a) If $X^{\prime}$ is an optimal $X$ for $\tau$ in (5), then (i) $\tau$ is an optimal $\rho$ for $X^{\prime}$ in (6), and (ii) all members of an optimal realising ensemble for $\tau$ are optimal $\rho$ for $X^{\prime}$ in (4). (b) If $\rho^{\prime}$ is an optimal $\rho$ for $Y$ in (6), then $Y$ is an optimal $X$ for $\rho^{\prime}$ in (5).

Proof. Statement (a)(i) is proven by inserting (6) in (5) and exploiting the premise that $X^{\prime}$ is an optimal $X$. This gives $\hat{f}(\tau)=\operatorname{Tr} \tau X^{\prime}-\max _{\rho}\left(\operatorname{Tr} \rho X^{\prime}-\hat{f}(\rho)\right)$. Putting $\rho=\tau$ yields an upper bound on the right-hand side because $\tau$ is not necessarily optimal in the maximisation. However, the value of the bound we obtain is $\hat{f}(\tau)$, which happens to be equal to the left-hand side. Thus this choice really is an optimal one, proving optimality of $\tau$ for $X^{\prime}$ in (6).

Statement (b) is proven similarly, by inserting (5) in (6).

Considering statement (a)(ii), let $\left\{\left(p_{i}, \tau_{i}\right)\right\}$ be an optimal ensemble for $\tau$ (with $p_{i}>0$ ). Thus $\hat{f}(\tau)=\sum_{i} p_{i} f\left(\tau_{i}\right)$. By assumption, $\hat{f}(\tau)=\operatorname{Tr} \tau X^{\prime}-$ $f^{*}\left(X^{\prime}\right)$. Inserting (4) and expanding unity as $\sum_{i} p_{i}$ yields $\sum_{i} p_{i} f\left(\tau_{i}\right)=\operatorname{Tr} \tau X^{\prime}-$ $\sum_{i} p_{i} \max _{\rho}\left(\operatorname{Tr} \rho X^{\prime}-f(\rho)\right)$. If we now replace $\rho$ by $\tau_{i}$ in the $i$-th summation term we get an upper bound on the right-hand side, with equality only if all the $\tau_{i}$

\footnotetext{
${ }^{2}$ For our purposes the Corollaries from 9 have to be restated with $\mathbb{R}^{n}$ replaced by $\mathcal{S}(\mathcal{H})$. This causes no problems if one extends the domain of $f$ to the affine space of all trace 1 Hermitian operators and defines $f(x)=+\infty$ for negative $x$.
} 
are optimal $\rho$ for $X^{\prime}$. The bound is easily seen to be $\sum_{i} p_{i} f\left(\tau_{i}\right)$, which is actually equal to the left-hand side. We find again that the bound is sharp, and optimality of the $\tau_{i}$ follows.

\section{Additivity}

These basic results will now prove to be a powerful tool for studying the additivity issue of the EoF. Let $\mathcal{H}_{I}$ and $\mathcal{H}_{I I}$ be two copies of the Hilbert space $\mathcal{H}_{A} \otimes \mathcal{H}_{B}$, and define $\mathcal{H}=\mathcal{H}_{I} \otimes \mathcal{H}_{I I}$. We will reserve the symbol $\otimes$ for tensor products with respect to the A-B subdivision, and the symbol $\otimes$ for tensor products regarding the I-II subdivision. Strong superadditivity of the EoF 4 is the inequality

$$
E_{F}(\rho) \geq ? E_{F}\left(\rho_{I}\right)+E_{F}\left(\rho_{I I}\right),
$$

for $\rho$ a state on $\mathcal{H}$, and $\rho_{I}$ and $\rho_{I I}$ its reductions to $\mathcal{H}_{I}$ and $\mathcal{H}_{I I}$, respectively.

The following Lemma is simple but crucial:

Lemma 1. For any bounded function $f$ defined on $\mathcal{S}(\mathcal{H})$, strong superadditivity of $\hat{f}$

$$
\hat{f}(\rho) \geq ? \hat{f}\left(\rho_{I}\right)+\hat{f}\left(\rho_{I I}\right),
$$

is equivalent to subadditivity of the conjugate function $f^{*}$ with respect to the Kronecker sum:

$$
f^{*}\left(X_{1} \otimes \mathbb{I}+\mathbb{I} \otimes X_{2}\right) \leq ? f^{*}\left(X_{1}\right)+f^{*}\left(X_{2}\right) .
$$

Proof. Set $Z=X_{1} \otimes \mathbb{I}+\mathbb{I} \otimes X_{2}$. Then, using (5) and assuming the validity of (9) yields

$$
\begin{aligned}
\hat{f}(\rho) & =\sup _{X} \operatorname{Tr}[\rho X]-f^{*}(X) \\
& \geq \sup _{X_{1}, X_{2}} \operatorname{Tr}[\rho Z]-f^{*}(Z) \\
& \geq \sup _{X_{1}, X_{2}} \operatorname{Tr}\left[\rho_{I} X_{1}+\rho_{I I} X_{2}\right]-f^{*}\left(X_{1}\right)-f^{*}\left(X_{2}\right) \\
& =\hat{f}\left(\rho_{I}\right)+\hat{f}\left(\rho_{I I}\right),
\end{aligned}
$$

which is (8).

The converse follows from (6). Assuming the validity of (8) yields

$$
\begin{aligned}
f^{*}(Z) & =\max _{\rho} \operatorname{Tr}[\rho Z]-\hat{f}(\rho) \\
& \leq \max _{\rho} \operatorname{Tr}\left[\rho_{I} X_{1}+\rho_{I I} X_{2}\right]-\hat{f}\left(\rho_{I}\right)-\hat{f}\left(\rho_{I I}\right) \\
& =\max _{\rho_{1}, \rho_{2}} \operatorname{Tr}\left[\rho_{1} X_{1}+\rho_{2} X_{2}\right]-\hat{f}\left(\rho_{1}\right)-\hat{f}\left(\rho_{2}\right) \\
& =f^{*}(X)+f^{*}(Y),
\end{aligned}
$$

which is (9).

The appearance of the Kronecker sum in Lemma 1 suggests that the consideration of the function $f^{*} \circ \log$ is a more natural setting for studying additivity. Defining $g:=f^{*} \circ \log$ and setting $X_{i}=\log M_{i}$, (9) becomes

$$
g\left(M_{1} \otimes M_{2}\right) \leq ? g\left(M_{1}\right)+g\left(M_{2}\right)
$$


for $M_{1}, M_{2} \in \mathcal{B}^{+}(\mathcal{H})$. Restating (5) and (6) in terms of $M$, we have

$$
\begin{aligned}
g(M) & =\max _{\rho \in \mathcal{S}(\mathcal{H})} \operatorname{Tr}[\rho \log (M)]-f(\rho) \\
\hat{f}(\rho) & =\max _{M \in \mathcal{B}^{+}(\mathcal{H})} \operatorname{Tr}[\rho \log (M)]-g(M) .
\end{aligned}
$$

Strictly speaking, these quantities are defined only for positive $M$. However, when $M$ is singular, we can still make sense out of it by the usual extension $\operatorname{Tr}[\rho \log (M)]=-\infty$ for any $\rho$ that is not completely supported on the range of $M$.

We can now restate Lemma 1 in the form of a Theorem, which is our first main result:

Theorem 1. For any function $f$ defined on $\mathcal{S}(\mathcal{H})$, and with $g$ defined on $\mathcal{B}^{+}(\mathcal{H})$ by (10), strong superadditivity of the convex closure $\hat{f}$,

$$
\hat{f}(\rho) \geq ? \hat{f}\left(\rho_{I}\right)+\hat{f}\left(\rho_{I I}\right),
$$

is equivalent to subadditivity of $g$,

$$
g\left(M_{1} \otimes M_{2}\right) \leq ? g\left(M_{1}\right)+g\left(M_{2}\right) .
$$

Note that the expression $\operatorname{Tr}[\rho \log (M)]-g(M)$ is invariant under multiplication of $M$ by a positive scalar. Hence, one could impose the restriction $\operatorname{Tr} M=1$, i.e. that $M$ should be a state, or alternatively $M \leq \mathbb{I}$, which is what we shall do.

An immediate corollary of this Theorem is the equivalence of the strong superadditivity of the EoF with the subadditivity of $g=E^{*} \circ \log$, where $E^{*}$ is the conjugate of the entanglement functional $E(\rho)=S\left(\operatorname{Tr}_{A} \rho\right)$. We have chosen to present Theorem 1 in the more general way because it obviates the rather remarkable independence of the Theorem on any property of the function $f$ at all. Specifically, while for the sake of defining the EoF it is necessary to split up the Hilbert space into two parties A and B, this is something the Theorem is completely oblivious of.

The only interesting feature of $E$ we can exploit at this level is its concavity. Concavity allows to simplify the conjugation expression by replacing the maximisation over all mixed states by a maximisation over pure states. Indeed, the argument of the maximisation in

$$
g(M)=\max _{\rho \in \mathcal{S}(\mathcal{H})} \operatorname{Tr}[\rho \log (M)]-E(\rho)
$$

is a convex function of $\rho$, and it is well-known [9] that a convex function achieves its maximum over a convex set always in an extreme point of that set, in this case in a pure state. Thus:

$$
g(M)=\max _{\psi \in \mathcal{H}} \operatorname{Tr}[\Psi \log (M)]-E(\Psi) .
$$

Theorem 1 reduces the additivity problem for the convex closure, originally defined as a minimisation over ensembles, to an equivalent problem for the conjugate function, defined as a maximisation over pure states. If counterexamples are found for (13), this automatically disproves strong superadditivity (12), so this simplification does not come at the cost of reduced power. Specifically, by "inverting" the proof of Lemma 1 (or Theorem 1) and employing Proposition 1, we easily get the following: 
Proposition 2. If $\rho$ violates strong superadditivity of $\hat{f}$, [12), $M_{1}$ is optimal for $\rho_{I}$ in (11), and $M_{2}$ is optimal for $\rho_{I I}$, then $M_{1} \otimes M_{2}$ violates subadditivity of $g$ (13). If $M_{1} \otimes M_{2}$ violates (13) and $\rho$ is optimal for $M_{1} \otimes M_{2}$ in (10), then $\rho$ violates (12).

\section{Maximal Output Purity}

Exploiting Theorem 1, we will now show that strong superadditivity of $E_{F}$ would follow as a consequence of another additivity conjecture, concerning quantum channel capacities. Recollect that, since $E$ is concave, the optimal $\rho$ in (4) will be an extreme point of the feasible set, i.e. a pure state, so:

$$
E^{*}(X)=\max _{\psi \in \mathcal{H}} \operatorname{Tr}[\Psi X]-E(\Psi) .
$$

From the additivity of $E$ over pure states it easily follows that the corresponding function $g=E^{*} \circ \log$ is superadditive, hence subadditivity of $g$ implies its additivity.

5.1. Step 1. The maximisation in $g$ can be rewritten in terms of a maximal eigenvalue $\lambda_{\max }$ :

Lemma 2. For any $M \in \mathcal{B}^{+}(\mathcal{H})$,

$$
\begin{aligned}
g(M) & :=\max _{\psi}\left(\operatorname{Tr}[\Psi \log M]-S\left(\operatorname{Tr}_{A} \Psi\right)\right) \\
& =\max _{\tau \in \mathcal{S}\left(\mathcal{H}_{B}\right)} \lambda_{\max }\left(\log M+\log \left(\mathbb{I}_{A} \otimes \tau\right)\right) .
\end{aligned}
$$

Note that we will henceforth consider $\log M+\log \left(\mathbb{I}_{A} \otimes \tau\right)$ as an operator restricted to the range intersection $\operatorname{ran}(M) \cap \operatorname{ran}(\mathbb{I} \otimes \tau)$.

Proof.

$$
\begin{aligned}
& \max _{\tau} \lambda_{\max }\left(\log M+\log \mathbb{I}_{A} \otimes \tau\right) \\
= & \max _{\tau} \max _{\psi} \operatorname{Tr}\left[\Psi\left(\log M+\log \mathbb{I}_{A} \otimes \tau\right)\right] \\
= & \max _{\tau} \max _{\psi} \operatorname{Tr}[\Psi \log M]+\operatorname{Tr}\left[\operatorname{Tr}_{A}(\Psi) \log \tau\right] \\
= & \max _{\psi} \operatorname{Tr}[\Psi \log M]-S\left(\operatorname{Tr}_{A} \Psi\right) .
\end{aligned}
$$

In step (16) we have used the Rayleigh-Ritz representation of a maximal eigenvalue, and in step (17) we have used the fact that relative entropy is non-negative and attains the value zero when (and only when) its arguments are equal. Specifically:

$$
\begin{aligned}
0 & =\min _{\tau} S(\rho \| \tau) \\
& =\min _{\tau}-S(\rho)-\operatorname{Tr}[\rho \log \tau] \\
& =-S(\rho)-\max _{\tau} \operatorname{Tr}[\rho \log \tau] .
\end{aligned}
$$


5.2. Step 2. Using the Lie-Trotter formula, the logarithm can be replaced by a limit of a power function.

\section{Lemma 3.}

$$
\exp g(M)=\lim _{p \rightarrow 0} h_{p}^{1 / p}(M)
$$

where

$$
h_{p}(M):=\max _{\tau}\left\|M^{p / 2}(\mathbb{I} \otimes \tau)^{p} M^{p / 2}\right\|
$$

and $\|$.$\| denotes the operator norm.$

Proof. Taking the exponential of both sides of (15) and noting $\exp \lambda_{\max }(M)=$ $\lambda_{\max } \exp (M)$, we get

$$
\exp g(M)=\max _{\tau}\|\exp (\log M+\log (\mathbb{I} \otimes \tau))\| .
$$

To make sense of this formula, we extend $\exp (\log M+\log (\mathbb{I} \otimes \tau))$ as 0 on the complement of $\operatorname{ran}(M) \cap \operatorname{ran}(\mathbb{I} \otimes \tau)$, as in [15]. The Lie-Trotter formula has a continuous version (see the remark after Lemma 3.3 in [15])

$$
\exp (A+B)=\lim _{p \rightarrow 0}(\exp (p A / 2) \exp (p B) \exp (p A / 2))^{1 / p} .
$$

In particular, this gives us

$$
\exp (\log M+\log (\mathbb{I} \otimes \tau))=\lim _{p \rightarrow 0}\left(M^{p / 2}(\mathbb{I} \otimes \tau)^{p} M^{p / 2}\right)^{1 / p} .
$$

Define the shorthand functions

$$
\begin{aligned}
f(\tau) & :=\|\exp (\log M+\log (\mathbb{I} \otimes \tau))\| \\
f_{p}(\tau) & :=\left\|\left(M^{p / 2}(\mathbb{I} \otimes \tau)^{p} M^{p / 2}\right)^{1 / p}\right\|
\end{aligned}
$$

over $\mathcal{S}(\mathcal{H})$. By $\left[18\right.$ and the triangle inequality for norms, $f_{p}$ converges pointwise to $f$. The functions $f_{p}$ are clearly continuous for $p>0$. By Lemma 4.1 of [15], $f$ is continuous too. From [16] (p. 118) we have that $f_{p}$ decreases monotonously to $f$ as $p$ decreases to 0 . The set $\mathcal{S}(\mathcal{H})$, over which $f$ and $f_{p}$ are defined, is compact. Hence, all the prerequisites are fulfilled to apply Dini's theorem [17, and we get that the convergence of $f_{p}$ to $f$ is uniform over $\mathcal{S}(\mathcal{H})$.

Finally, uniform convergence is equivalent with convergence in the sup-norm. By the triangle inequality for norms, that in turn implies that the sup-norm of $f_{p}$ converges to the sup-norm of $f$. Therefore, $h_{p}^{1 / p}(M)=\max _{\tau} f_{p}(\tau)=\left\|f_{p}\right\|_{\mathcal{S}}$ converges to $\|f\|_{\mathcal{S}}=\max _{\tau} f(\tau)=\exp g(M)$.

Additivity of $g$ would thus follow as a consequence of multiplicativity of $h_{p}$, $h_{p}\left(M_{1} \otimes M_{2}\right)=$ ? $h_{p}\left(M_{1}\right) h_{p}\left(M_{2}\right)$, for $p \downarrow 0$. Following [18, we say that a property holds for $p \downarrow a$ if it holds for an arbitrarily small, but finite, interval $p \in(a, a+\epsilon]$, $\epsilon>0$. 
5.3. Step 3. The quantity $h_{p}(M)$ is formally equal to the maximal output purity 18, 19 20 of quantum filtering operations. Indeed,

$$
\begin{aligned}
h_{p}(M) & =\max _{\tau, \phi} \operatorname{Tr}\left[\Phi\left(M^{p / 2}(\mathbb{I} \otimes \tau)^{p} M^{p / 2}\right)\right] \\
& =\max _{\tau, \phi} \operatorname{Tr}\left[\tau^{p} \operatorname{Tr}_{A}\left[M^{p / 2} \Phi M^{p / 2}\right]\right] \\
& =\max _{\phi}\left\|\operatorname{Tr}_{A}\left[M^{p / 2} \Phi M^{p / 2}\right]\right\|_{q} \\
& =\nu_{q}(\Lambda),
\end{aligned}
$$

where $q=1 /(1-p)$ and $\|.\|_{q}$ denotes the Schatten $q$-norm [21], and $\nu_{q}(\Lambda)$ is the maximal output purity measured by the Schatten $q$-norm of the (non-trace preserving) operation

$$
\Lambda: \rho \mapsto \Lambda(\rho)=\operatorname{Tr}_{A}\left[M^{p / 2} \rho M^{p / 2}\right] .
$$

If this operation would be trace preserving, we would call it a channel.

5.4. Step 4. We now claim that there is no advantage in restricting attention to operations of the form (19). It is of course true that the class of operations (19) is rather specific. They admit a Kraus representation such that the block column matrix $\left(A_{i}\right)_{i}$ obtained by stacking the Kraus element matrices $A_{i}$ vertically, equals $M^{p / 2}$, which is a positive matrix. Necessary conditions are that $\sum_{i} A_{i}^{\dagger} A_{i}=M^{p}$ (which is $\left.\leq \mathbb{I}\right)$ and the input dimension of the operation should equal the output dimension times the number of elements.

However, as regards the maximal output purity question, these structural peculiarities offer no additional mileage. To see this, consider the specific case that $M$ is a partial isometry $M=U \Sigma U^{\dagger}$, where $\Sigma=|1\rangle\langle 1| \otimes \mathbb{I}_{B}$ and $U$ is any unitary, then

$$
\begin{aligned}
\nu_{q}(\Lambda) & =\max _{\phi \in \mathcal{H}}\left\|\operatorname{Tr}_{A}\left[U \Sigma^{p / 2} U^{\dagger} \Phi U \Sigma^{p / 2} U^{\dagger}\right]\right\|_{q} \\
& =\max _{\phi^{\prime} \in \mathcal{H}}\left\|\operatorname{Tr}_{A}\left[U \Sigma^{p / 2} \Phi^{\prime} \Sigma^{p / 2} U^{\dagger}\right]\right\|_{q} \\
& =\max _{\phi^{\prime \prime} \in \mathcal{H}_{B}}\left\|\operatorname{Tr}_{A}\left[U\left(|1\rangle\langle 1| \otimes \Phi^{\prime \prime}\right) U^{\dagger}\right]\right\|_{q},
\end{aligned}
$$

which is the generic case for operations from $\mathcal{H}_{A}$ to $\mathcal{H}_{A}$. Thus, the case for the "special operations" $\mathcal{H} \mapsto \mathcal{H}_{A}$ contains the generic $\mathcal{H}_{A} \mapsto \mathcal{H}_{A}$ case and is therefore not easier to prove.

5.5. Step 5. The exponent $p$ of $M$, occurring in $\Lambda$, is coupled to $q$, occurring in $\nu_{q}$, via the relation $q=1 /(1-p)$. To cap off our argument, we "decouple" $p$ and $q$ by replacing $M^{p / 2}$ with a general matrix $0 \leq X \leq \mathbb{I}$, strenghtening our multiplicativity conjecture ever so slightly. Noting finally that $p \downarrow 0$ corresponds to $q \downarrow 1$, we get our second main result:

Theorem 2. If $\nu_{q}(\Lambda)$ is multiplicative for $q \downarrow 1$ and for any filtering operation $\Lambda$, then the entanglement of formation is strongly subadditive. 
Multiplicativity of $\nu_{q}$ had been conjectured in [18 for trace preserving channels. It has been proven for entanglement breaking channels [20, unital qubit maps 22 and depolarising channels 23, but, unfortunately, was refuted in [19] for $q>4.79$. Nevertheless, the conjecture might still be true for $q \downarrow 1$.

Theorem 2 has to be compared to the main technical result in [7], which states that additivity of the Holevo capacity for given channels implies additivity of the EoF for certain states. In a sense, our Theorem 2 is stronger because we get the stronger outcome of strong subadditivity. On the other hand, this comes at the price of having to consider non-trace-preserving operations.

After the appearance of the first draft of this manuscript, Shor proved [24] the equivalence of four additivity conjectures: strong superadditivity of the EoF, ordinary additivity of the EoF, additivity of the maximal output purity $\nu_{S}$ of a channel as measured by the entropy, and additivity of the classical (Holevo) capacity of a channel. As multiplicativity of $\nu_{q}(\Lambda)$ for $q \downarrow 1$ implies additivity of $\nu_{S}(\Lambda)$ [18, Shor's third equivalence provides an alternative proof for our result Theorem 2.

\section{Conclusion}

In conclusion, we have shown how a simple convex analytical argument leads to a simpler formulation of the entanglement of formation and an especially simple equivalent condition for strong superadditivity of the EoF. Based on this we have found the second result that strong superadditivity of the EoF would follow as a consequence of the multiplicativity of the maximum output purity $\nu_{q}$ of quantum filtering operations, for $q \downarrow 1$.

Acknowledgement. We gratefully acknowledge comments by M.B. Plenio, J. Eisert, M.B. Ruskai and Ch. King. SLB currently holds a Wolfson-Royal Society Research Merit Award.

\section{References}

1. P.M. Hayden, M. Horodecki and B.M. Terhal, J. Phys. A 34(35):6891-6898 (2001).

2. W. Wootters, Phys. Rev. Lett. 80, 2245 (1998).

3. F. Benatti and H. Narnhofer, Phys. Rev. A 63, 042306 (2001).

4. KG.H. Vollbrecht and R.F. Werner, Phys. Rev. A 64, 062307 (2001).

5. G. Vidal, W. Dür and J.I. Cirac, Phys. Rev. Lett. 89, 027901 (2002).

6. M. Horodecki, A. Sen De and U. Sen, quant-ph/0207031 (2002).

7. K. Matsumoto, T. Shimono and A. Winter, quant-ph/0206148 (2002).

8. Heng Fan, quant-ph/0210169 (2002).

9. R.T. Rockafellar, Convex Analysis, Princeton University Press, Princeton (1970).

10. S. Boyd and L. Vandenberghe, Convex Optimization, available online at http://www.stanford.edu/ boyd/cvxbook.html (2002).

11. C.H. Bennett, D.P. DiVincenzo, J. Smolin and W.K. Wootters, Phys. Rev. A 54, 3824 (1996).

12. R.B. Lockhart, J. Math. Phys. 41(10):6766-6771 (2000).

13. K.M.R. Audenaert, F. Verstraete and B. DeMoor, Phys. Rev. A 64, 052304 (2001).

14. A. Uhlmann, quant-ph/9704017 (1997).

15. F. Hiai and D. Petz, Lin. Alg. Appl. 181, 153-185 (1993).

16. T. Ando and F. Hiai, Lin. Alg. Appl. 197, 198, 113-131 (1994).

17. T.M. Apostol, Mathematical Analysis, Addison-Wesley, (1974).

18. G.G. Amosov, A.S. Holevo and R.F. Werner, Problems in Information Transmission 36, 25-34 and math-ph/0003002 (2000).

19. R.F. Werner and A.S. Holevo, J. Math. Phys. 43(9), 4353-4357 (2002). 
20. C. King, quant-ph/0212057 (2002).

21. R.A. Horn and C.R. Johnson, Topics in Matrix Analysis, Cambridge University Press, Cambridge (1991).

22. C. King, J. Math. Phys. 43(9), 4334-4340 (2002).

23. C. King, quant-ph/0204172 (2002).

24. P.W. Shor, quant-ph/0305035 (2003). 